\title{
Knowledge Mapping Analysis of International Research on Acupuncture for Low Back Pain Using Bibliometrics
}

\author{
Haotian Pan (1D) \\ Ziqi Xi $\left.{ }^{\prime}\right)^{\prime}$ \\ Xintong Yu' \\ Xuqiu Sun ${ }^{2}$ \\ Xuqiang Wei ${ }^{1}$ \\ Ke Wang $\mathbb{D}^{1,2}$
}

'Acupuncture Anesthesia Clinical Research Institute, Yueyang Hospital of Integrated Traditional Chinese and Western Medicine, Shanghai University of Traditional Chinese Medicine, Shanghai, People's Republic of China; ${ }^{2}$ Office of National Clinical Research Base of TCM, Yueyang Hospital of Integrated

Traditional Chinese and Western Medicine, Shanghai University of Traditional Chinese Medicine, Shanghai, People's Republic of China
Correspondence: Ke Wang Acupuncture Anesthesia Clinical Research Institute, Yueyang Hospital of Integrated Traditional Chinese and Western Medicine, Shanghai University of Traditional Chinese Medicine, Shanghai, 200437, People's Republic of China Email wangke8430@I63.com
Objective: Acupuncture has made recommendations to relieve low back pain (LBP) in a few guidelines. However, few articles focused on the papers published before, which may reflect the trend of study, main researchers, reciprocal collaboration, and other information in this field. In this study, we utilize the bibliometric analysis methods to objectively analyze the current trend and research hotspots about this field, aiming to provide a foundation and reference for future study.

Methods: The Web of Science database was searched for publications related to acupuncture therapy for treating low back pain between 1985 and 2021. VOSviewer and CiteSpace were used to analyze the annual publication, authors and co-cited authors and their countries (regions) and institutions, journals and co-cited journals, co-cited references, co-occurrence keywords, burst keywords, and the relevant centrality.

Results: A total of 1279 papers were retrieved from the database, and the trend of annual publications maintained growth. Article was the most document type (867). The USA (451) and the Kyung Hee University (49) were the most productive country (region) and institution, respectively, while the USA (0.37) and University of Maryland (0.13) had the highest centrality. MacPherson, Hugh and Sherman, Karen J. published the most papers in this field (32), and Ernst, Edzard became the most influential author (474 co-citations). Journal of Alternative and Complementary Medicine was the most productive journal (79), whereas SPINE was the most influential one (3111 co-citations). Haake's (2007) article had the most co-citation count (186), while Furlan's (2005) article had the highest centrality (0.23). The three frontier topics were pain management, disease, and protocol.

Conclusion: The present study investigated publications on acupuncture for treating low back pain using bibliometric analysis methods, which may help researchers explore the potential directions for the future.

Keywords: low back pain, acupuncture, bibliometric analysis, VOSviewer, CiteSpace

\section{Introduction}

Acupuncture plays a crucial role in Traditional Chinese Medicine (TCM) and has been regarded as the treasure of China. It is characterized by the insertion of solid and stainless steel needles into specific acupoints. ${ }^{1}$ Based on the Web of Science database and PubMed, respectively, Ma et al and Lee et al have conducted two global bibliometric analyses to investigate the development of acupuncture in both preclinical and clinical fields. ${ }^{2,3}$ Nowadays, acupuncture has been used as a complementary therapy in treating various diseases, especially for pain management. In the National Institute for Health and Clinical Excellence (NICE) guideline 
updated recently (NG193), acupuncture has been added into the recommendations of management for chronic pain (www.nice.org.uk/guidance/ng193). According to the International Association for the Study of Pain (IASP), chronic pain refers to pain that persists for more than three months. ${ }^{4}$ Low back pain (LBP) is defined as pain in the area between the lower rib margins and the buttock creases. ${ }^{5,6}$ It can be segmented into acute $(<6$ weeks), subacute (6-12 weeks), and chronic ( $>12$ weeks) low back pain, according to the duration of onset by the American College of Physicians (ACP) clinical practice guideline. ${ }^{7}$ Acupuncture has received increasing attention in treating LBP, a growing body of literature has emerged rapidly. As recommended by the 2016 CDC Guidelines for Prescribing Opioids for Chronic Pain and the 2017 ACP clinical practice guideline, acupuncture has been one of the first choices for treating LBP. ${ }^{7,8}$ However, little attention has been paid to topic hot spots and trends in acupuncture for LBP.

LBP has become the leading cause of disability worldwide. ${ }^{5,9}$ As shown in the reports of Global Burden of Disease (GBD) 2019 (http://www.healthdata.org/results/ gbd_summaries/2019), the prevalence of LBP is approximately $7 \%$ in all age groups around the world, while females have a higher prevalence and incidence. Generally, the episodes of LBP are short-lasting, but recurrence is very common. ${ }^{5}$ A cohort study including 250 patients with a 12-month follow-up showed that $69 \%$ of individuals had a recurrence within 12 months, and the awkward posture, long time sitting, and more than two previous episodes are the three most common inducements of recurrence. ${ }^{10}$ As a complex condition that consists of psychological, social, and biophysical factors, nonpharmacological approaches, including acupuncture, have been proposed as first-line treatments for LBP. ${ }^{11}$

Bibliometric analysis is a highly important tool for comprehensively recognizing the research advances in a specific field of scientific data, which can measure the interrelationships and impacts of publications through a series of mathematical and statistical tools, which has been implemented in many research fields. ${ }^{12,13}$ Hitherto, VOSviewer and CiteSpace are two widely used applications based on Java. Thus, in this study, VOSviewer and CiteSpace will be administrated to explore and visualize the scientific publications of acupuncture treatment research for LBP based on the Web of Science database.

\section{Materials and Methods}

\section{Source and Search Strategy}

The data were extracted from the Science Citation Index Expanded (SCI-expanded) of the Web of Science Core Collection database. The data retrieval strategy was set as topic: (acupuncture) AND topic: (low back pain); Timespan: 1985-2021 (retrieved date June 6, 2021). There were no restrictions on language, type of document, and type of research including. Only the repeated publications were excluded from the study.

\section{Analysis}

Our analysis included the following: (1) descriptive statistical analysis, descriptive analysis of the annual publication volume, authors, countries, institutions, and journals; (2) co-cited analysis, a co-cited map for authors, journals, and references: (3) co-occurrence analysis, a cooccurrence map for keywords; (4) burst keywords.

\section{Analysis Tool}

VOSviewer is a software tool for creating maps based on network data and for visualizing and exploring these maps. It can construct co-authorship, co-occurrence, citation, bibliographic coupling, or co-citation networks of scientific publications. ${ }^{14,15}$ Thus, the VOSviewer (version 1.6.16) was used to explore the existing network patterns, and identify and visualize countries (regions), institutions, authors and co-cited authors, journals and cocited journals, co-cited references, and co-occurrence keywords. The different nodes represent different items while the size of the circle, determined by the weight of the item, reflects the productivity. Lines between items represent links. Thicker lines mean stronger links. For building a visualizing network, the number of items in each research topic was limited according to their respective condition. CiteSpace is another computational tool designed to help researchers better understand the research fields they are engaged in. The centrality analysis and burst detection functions have received much attention from scholars in recent years. ${ }^{16,17}$ High centrality is often well recognized as a turning point or pivotal point in a field. ${ }^{13}$ In this study, CiteSpace (version 5.7. R5) was used to calculate the centralities between institutions, co-cited journals, co-cited authors, co-cited references and co-occurrence keywords, and the burst keywords. 


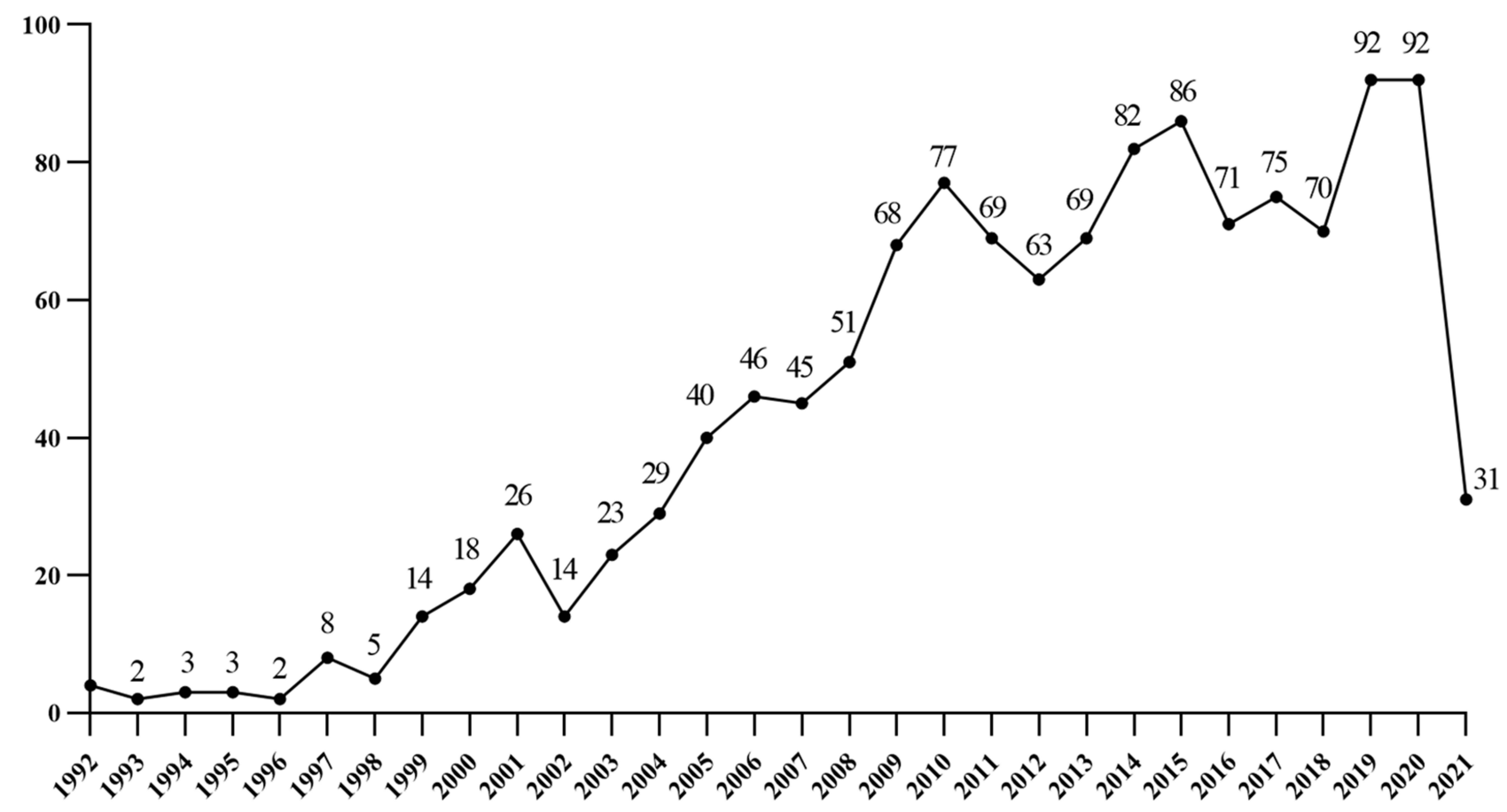

Figure I The number of annual publications on acupuncture treatment for LBP indexed by SCI-E. Abbreviations: LBP, low back pain; SCI-E, Science Citation Index-Expanded.

\section{Results}

\section{Analysis of Annual Publications and Document Type}

A total of 1279 publications were used in this study. The number of articles published each year is shown in Figure 1, and the first article was published in 1992. 1992 to 1996 could be seen as the first phase, and its development trend was steady and barely growing. 1997 to 2010 could be seen as the second phase, which showed an obvious increase. 2011 to 2020 could be seen as the third phase, which grew slowly and showed fluctuations, and reached the peak in 2019 and 2020. Table 1 enumerates the top 5 document types of these retrieved papers. Amid these types, the Article accounted for $67.787 \%$ and was followed by the Review of $22.83 \%$, as well as the

Table I Top 5 Document Types for Documents on Acupuncture for LBP

\begin{tabular}{|l|l|l|l|}
\hline Rangking & Type & Counts & \% of I 279 \\
\hline I & Article & 867 & 67.787 \\
2 & Review & 292 & 22.830 \\
3 & Editorial material & 50 & 3.909 \\
4 & Letter & 39 & 3.049 \\
5 & Meeting abstract & 18 & 1.407 \\
\hline
\end{tabular}

Abbreviation: LBP, low back pain.
Editorial material of $3.909 \%$. The Letter and Meeting abstract also had the percent of $3.049 \%$ and $1.407 \%$, respectively.

\section{Analysis of Country/Region}

Overall, the 1279 references were published by 58 countries or regions. For better visualization, 17 countries or regions with more than 15 articles were selected via VOSviewer (Figure 2). The top 5 countries/regions are stated in Table 2, and the United States of America (USA) had the most publications (451), followed by the People's Republic of China, England, Germany, and South Korea. Furthermore, The USA possessed the highest centrality (0.37), which may demonstrate the most relevant studies and cooperation with others. China had a centrality of 0.23 , indicating that the study of acupuncture therapy for LBP was also fashionable in China.

\section{Analysis of Institution}

A total of 1570 research institutions were involved, and 25 institutions with more than 15 papers were chosen for visualization (Figure 3 ). The top five institutions are listed in Table 3, of which Kyung Hee University had the most research articles on acupuncture for LBP (49). The other four institutions of the top five are Korea Institute of Oriental Medicine, Harvard University, 


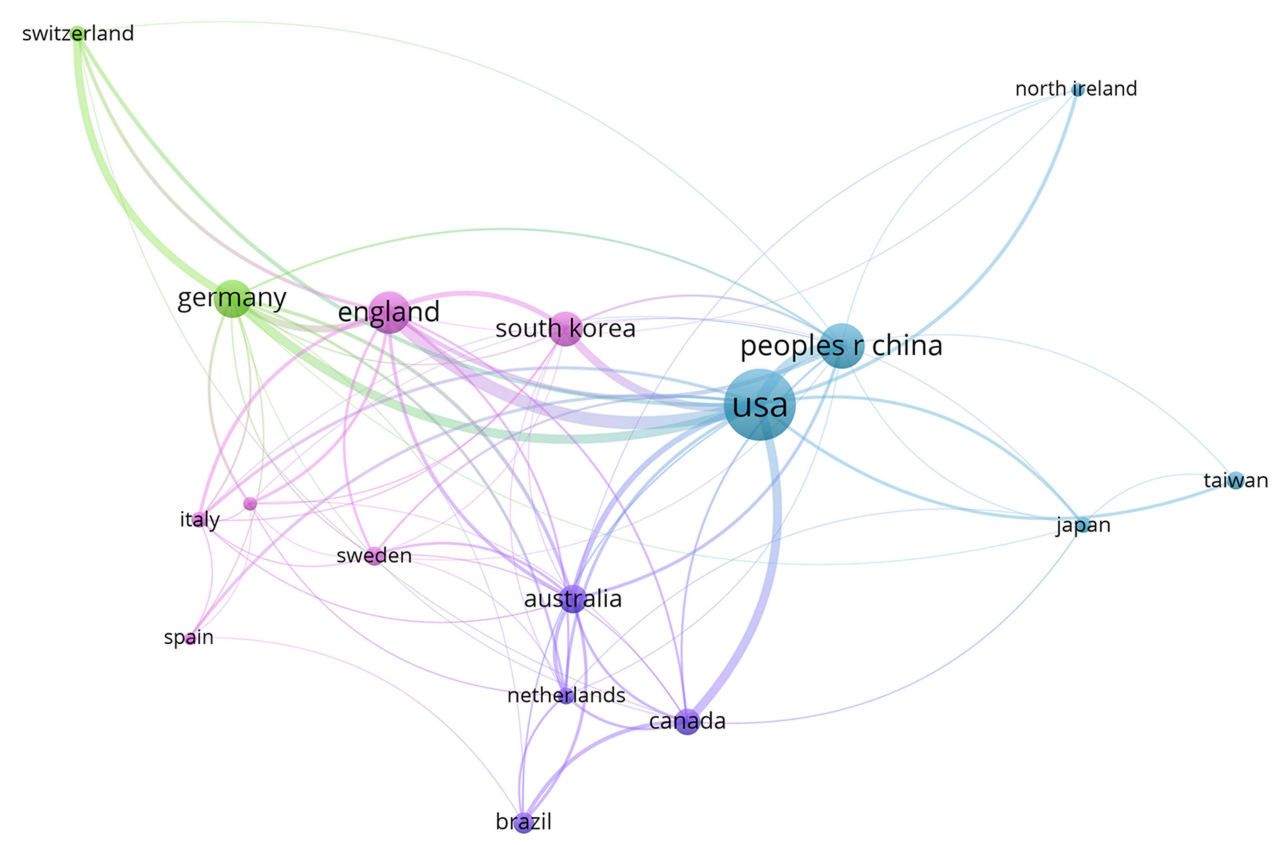

Figure 2 Map of active countries/regions on acupuncture treatment research for LBP. Abbreviation: LBP, low back pain.

University of Maryland, and University of York, in turn. Figure 4 displays the change of institutions in terms of time. Harvard University and the University of Maryland initiated the relevant study in early time, while the trend developed in Asia after 2015, represented by Beijing University of Chinese Medicine and Kyung Hee University. As a representative of a new emerging institution, Harvard Medical School also has published 31 papers until now.

\section{Analysis of Author and Co-Cited Author}

A total of 4760 authors contributed to these articles, we selected 108 ones with more than five papers for better visualization (Figure 5). The relationships between authors can also be observed in this map. In the top 10 authors listed in Table 4, MacPherson and Ernst come from England, Sherman, Kaptchuk, Witt, and Cherkin come from the USA, Ha, In-Hyuk, Lee, Myeong Soo, and Lee,
Jinho come from South Korea, Linde comes from Germany. The "year" in the Table means the time when the first article was published by each author. For instance, MacPherson published his first paper titled "Acupuncture for low back pain: traditional diagnosis and treatment of 148 patients in a clinical trial" in $2014 .^{18}$ This article focused on the diagnostic concordance among practitioners and individualized treatment of acupuncture for LBP.

Co-citation refers to the phenomenon that two or more authors or their articles are cited by other literature at the same time. Herein, we included 51 authors cited at least 50 times for visualization. As revealed in Figure 6 and Table 5, Ernst had the most co-cited frequency (474), but Deyo had the highest centrality (0.09). Cherkin, Furlan, Linde, and MacPherson also had relatively high frequency (>300), while Ernst, Kaptchuk, and Linde had comparatively higher centrality $(\geq 0.05)$.

Table 2 Top 5 Countries/Regions Performed Research on Acupuncture for LBP

\begin{tabular}{|l|l|l|l|l|}
\hline Ranking & Country/Region & Centrality & Counts & \% of I279 \\
\hline 1 & USA & 0.37 & $45 I$ & 35.262 \\
2 & Peoples R China & 0.23 & 180 & 14.073 \\
3 & England & 0.21 & 169 & 13.213 \\
4 & Germany & 0.13 & 137 & 10.711 \\
5 & South Korea & 0.02 & 117 & 9.148 \\
\hline
\end{tabular}

Abbreviation: LBP, low back pain. 


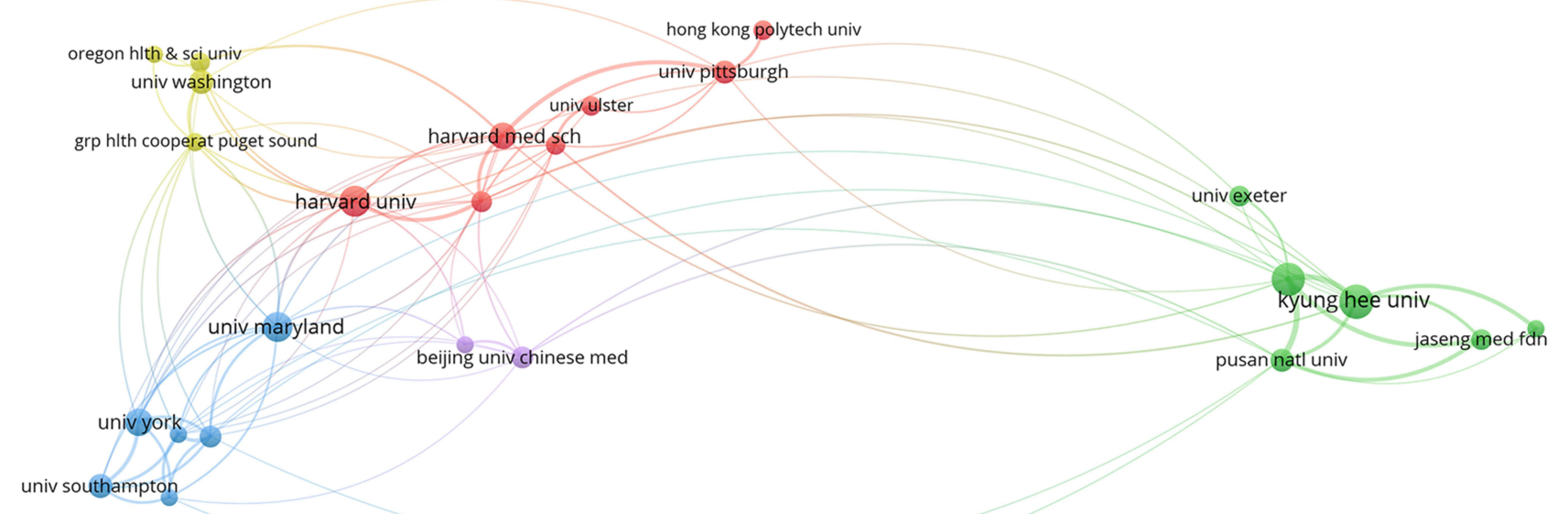

Figure 3 Map of active institutions on acupuncture treatment research for LBP. Abbreviation: LBP, low back pain.

\section{Analysis of Journal and Co-Cited Journal} There were 335 journals screened in this study, and finally, 30 journals producing more than 10 papers were selected for visualization (Figure 7). As enumerated in Table 6 , the first most productive journal was Journal of Alternative and Complementary Medicine, with 79 publications. According to the Journal Citation Reports (2020), the average IF of these top 10 journals is 3.65 .

Figure 8 presents the 61 co-cited journals at least 120 times. In this map, the distance between two journals in the visualization approximately indicates the relatedness of the journals in terms of co-citation links. In general, the closer two journals are located to each other, the stronger their relatedness. As shown in Table 7, SPINE had the most frequency (3111) and followed by PAIN, Journal of Alternative and Complementary Medicine, Cochrane Database of Systematic Reviews, Annals of Internal Medicine, Acupuncture in Medicine, and BMJ-British Medical Journal whose frequency are more than 1000 .

\section{Analysis of Co-Occurrence Keywords}

Co-occurrence refers to the phenomenon that two or more keywords appeared in other literature at the same time. In this study, we selected 198 keywords with more than ten times of co-occurrence for better visualization from a total of 3529 keywords (Figure 9). Table 8 lists the top 10 cooccurrence keywords. There was no doubt that "acupuncture" and "low back pain" were the two most frequent, with 692 and 644 publications, respectively. Moreover, all the other eight keywords had more than 100 frequencies. "Randomized controlled-trial" had the highest centrality (0.08), followed by "acupuncture" and "pain" with the centrality of 0.07 .

\section{Analysis of Co-Cited Reference}

A total of 34,174 references were generated from 1279 papers. Table 9 presents the top 10 co-cited references sorted by the co-citation counts. Of these ten references, six references were randomized controlled trials (RCTs), three were systematic review and meta-analysis, and one clinical guideline.

Table 3 Top 5 Institutions Performed Research on Acupuncture for LBP

\begin{tabular}{|l|l|l|l|l|l|}
\hline Ranking & Institution & Abbreviations & Centrality & Counts & \% of I279 \\
\hline I & Kyung Hee University & Kyung Hee Univ & 0.05 & 49 & 3.83 I \\
2 & Korea Institute of Oriental Medicine & Korea Inst Oriental Med & 0.04 & 45 & 3.518 \\
3 & Harvard University & Harvard Univ & 0.09 & 42 & 3.284 \\
4 & University of Maryland & Univ Maryland & 0.13 & 39 & 3.049 \\
5 & University of York & Univ York & 0.01 & 33 & 2.580 \\
\hline
\end{tabular}

Abbreviation: LBP, low back pain. 


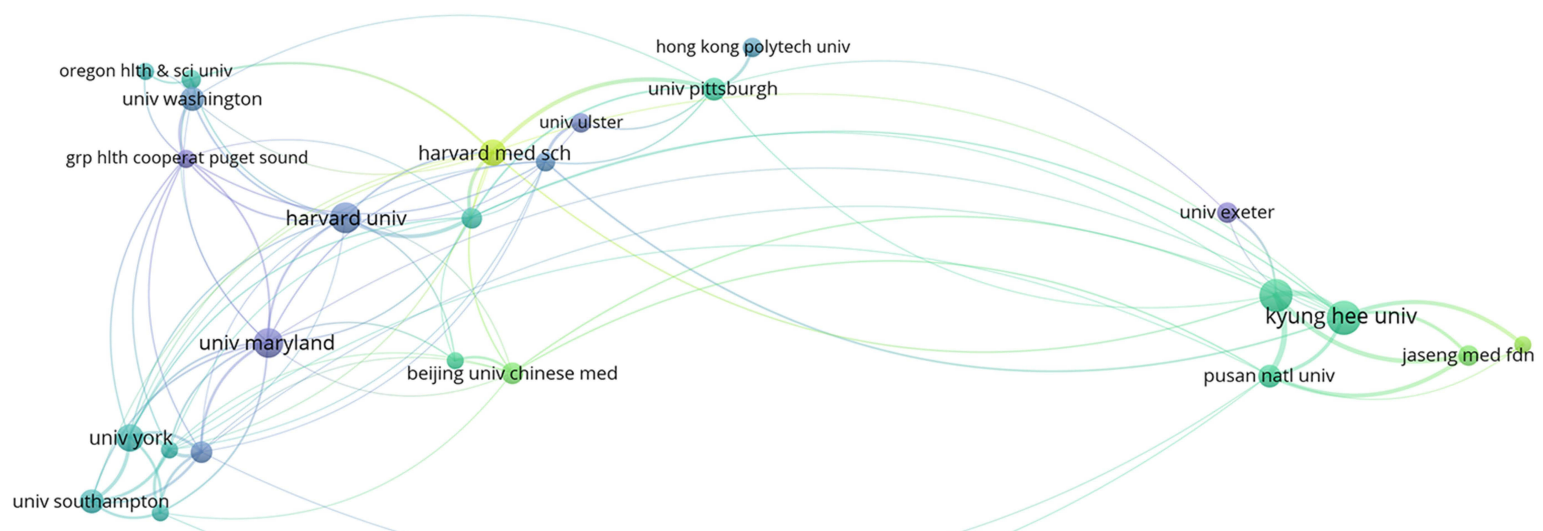

Figure 4 Map of active institutions on acupuncture treatment research for LBP in terms of time.

Abbreviations: LBP, low back pain.

liu, cun-zhi

deyo, ra

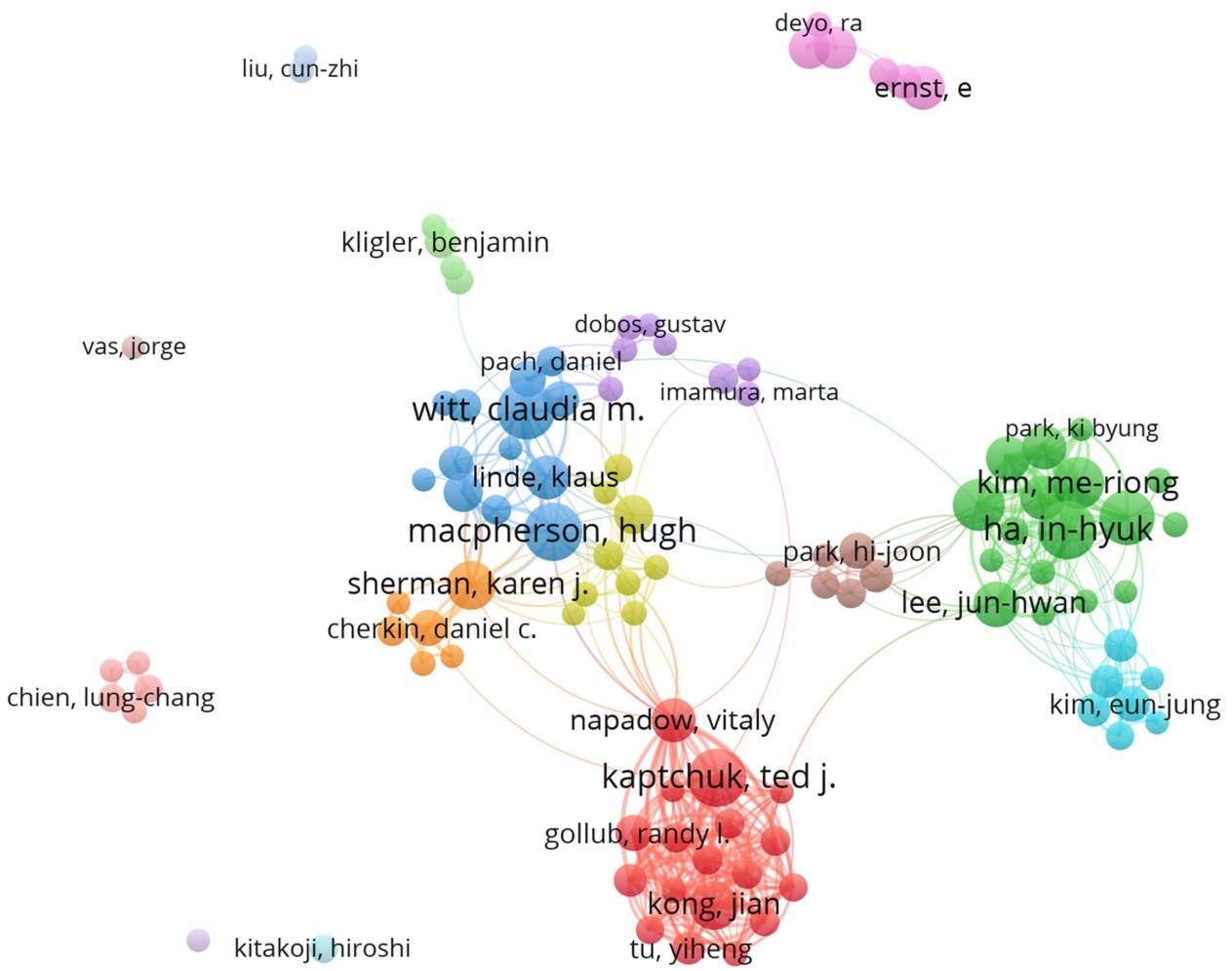

molsberger, a

macpherson, h.

Figure 5 Map of authors who study acupuncture treatment for LBP and their collaborations.

Abbreviation: LBP, low back pain. 
Table 4 Top 10 Authors Who Performed Research on Acupuncture for LBP

\begin{tabular}{|l|l|l|l|l|}
\hline Ranking & Author & Counts & Year* & Country \\
\hline 1 & MacPherson, Hugh & 32 & 2004 & England \\
2 & Sherman, Karen J. & 32 & 2001 & USA \\
3 & Kaptchuk, Ted J. & 28 & 2000 & USA \\
4 & Witt, Claudia M. & 27 & 2006 & USA \\
5 & Cherkin, Daniel C. & 25 & 1999 & USA \\
6 & Ha, In-Hyuk & 25 & 2010 & South Korea \\
7 & Ernst, Edzard & 22 & 1997 & England \\
8 & Lee, Myeong Soo & 22 & 2009 & South Korea \\
9 & Linde, Klaus & 22 & 2003 & Germany \\
10 & Lee, Jinho & 21 & 2013 & South Korea \\
\hline
\end{tabular}

Note: *The year when the first article published.

Abbreviation: LBP, low back pain.

The article published in 2007 by Haake had the most co-citation count (186). This article compared the effects of verum acupuncture, sham acupuncture, and guidelinebased conventional therapy for chronic LBP in a 6-month treatment, and deduced that the effectiveness of either verum or sham acupuncture was nearly twice than that of conventional therapy but there was no statistical difference of efficacy between verum and sham acupuncture ${ }^{19}$.
The article published in 2005 by Furlan had the highest centrality $(0.23)$. This systematic review collected papers about RCTs of acupuncture or dry-needling for nonspecific low back pain from 1996 to February 2003 and assessed the effect of these two interventions. The data suggested that in terms of pain relief and functional improvement, acupuncture was more effective than sham or no treatment immediately after treatment and in the short-term, and both acupuncture and dry-needling may be conducive adjuncts to other therapies for chronic LBP. ${ }^{20}$

\section{Analysis of Burst Keywords}

"Burst keywords" refers to keywords cited frequently over some time, thereby indicating the frontier areas. ${ }^{20}$ Figure 10 shows twenty burst keywords sorted by the "begin year". As displayed, the related investigation started from the keyword "rheumatoid arthritis" from 1992 and lasted until 2003. And then, "controlled trial" became popular among researchers from 1995 to 2005, with the highest strength (11.42) among these 20 burst keywords.

Currently, three keywords had become burst ones and last until now: pain management, disease, and protocol. It may indicate that researchers will focus more on the

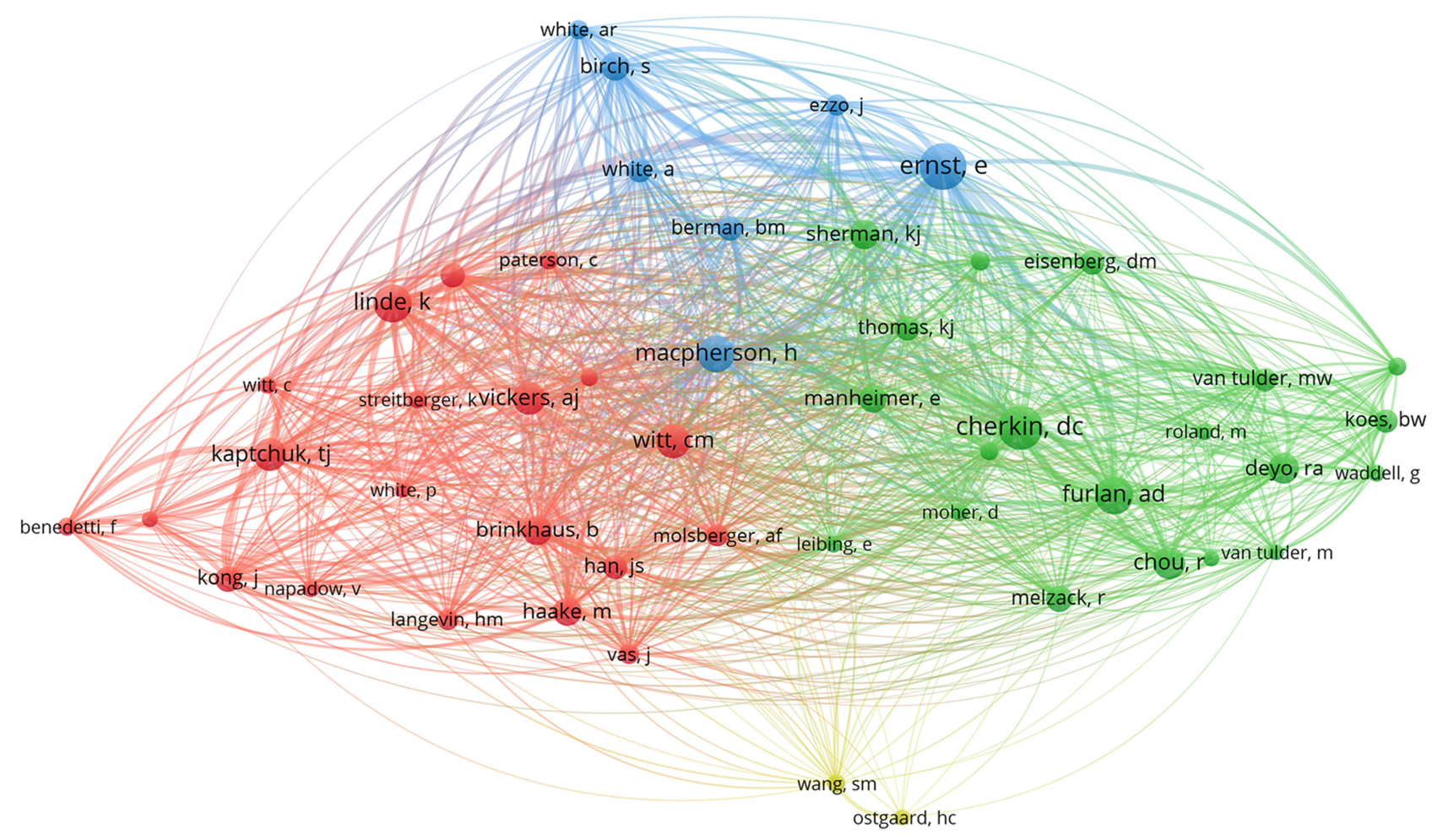

Figure 6 Map of co-cited authors who study acupuncture treatment for LBP. Abbreviation: LBP, low back pain. 
Table 5 Top 10 Co-Cited Authors Performed Research on Acupuncture for LBP

\begin{tabular}{|l|l|l|l|l|}
\hline Ranking & Author & Country & Frequency & Centrality \\
\hline 1 & Ernst, Edzard & England & 474 & 0.06 \\
2 & Cherkin, Daniel C. & USA & 429 & 0.04 \\
3 & Furlan, Andrea D. & Canada & 335 & 0.03 \\
4 & Linde, Klaus & Germany & 332 & 0.05 \\
5 & MacPherson, Hugh & England & 324 & 0.03 \\
6 & Witt, Claudia M. & USA & 282 & 0.02 \\
7 & Kaptchuk, Ted J. & USA & 265 & 0.06 \\
8 & Vickers, Andrew J. & USA & 256 & 0.04 \\
9 & Chou, Roger & USA & 245 & 0.03 \\
10 & Deyo, Richard A. & USA & 236 & 0.09 \\
\hline
\end{tabular}

Abbreviation: LBP, low back pain.

disease itself and make study protocols that pay more attention to pain management.

\section{Discussion}

Acupuncture is an effective and safe adjunct intervention for LBP, and it may be especially effective at chronic pain and improving motor function. ${ }^{29-31}$ The visual analog scale (VAS) score and Oswestry Disability Index (ODI) score are important indexes to judge the recovery of pain and functional activity and the curative effects after treatment for LBP. Studies have shown that acupuncture treatment could significantly improve in VAS and ODI scores for LBP, as well as a lower incidence of adverse events than other interventions (conventional medicines, routine care, and placebo). ${ }^{29,32}$ Furthermore, the brain regions such as default mode network (DMN), salience network (SN), and descending pain modulatory system (DPMS) are involved in acupuncture treatment for LBP $^{33}$ Kim et al have observed that acupuncture could enhance tactile acuity of pain-affected regions in chronic

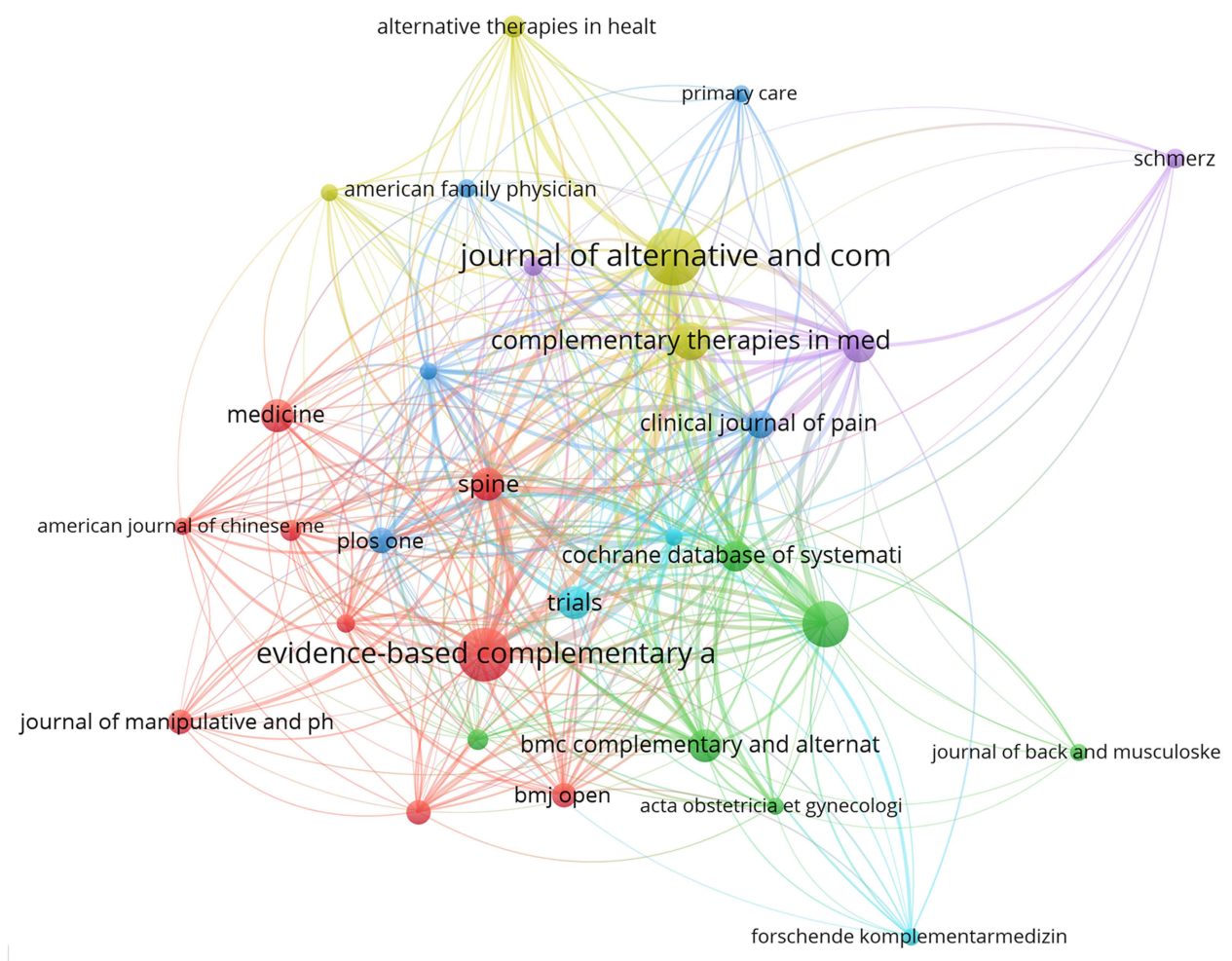

Figure 7 Map of active journals producing publications about acupuncture treatment for LBP. Abbreviation: LBP, low back pain. 
Table 6 Top 10 Relevant Journals on Acupuncture for LBP Research

\begin{tabular}{|c|c|c|c|c|c|}
\hline Ranking & Journal & Abbreviations & IF (Q)* & Counts & $\%$ of 1279 \\
\hline 1 & Journal of Alternative and Complementary Medicine & J Altern Complem Med & $2.6(2)$ & 79 & 6.182 \\
\hline 2 & $\begin{array}{l}\text { Evidence-based Complementary and Alternative } \\
\text { Medicine }\end{array}$ & $\begin{array}{l}\text { Evid Based Complement Alternat } \\
\text { Med }\end{array}$ & $2.629(3)$ & 69 & 5.399 \\
\hline 3 & Acupuncture in Medicine & Acupunct Med & $2.267(2)$ & 55 & 4.304 \\
\hline 4 & Complementary Therapies in Medicine & Complement Ther Med & $2.446(2)$ & 39 & 3.052 \\
\hline 5 & SPINE & Spine & $3.468(2)$ & 31 & 2.426 \\
\hline 6 & Trials & Trials & $2.279(3)$ & 31 & 2.426 \\
\hline 7 & BMC Complementary and Alternative Medicine & BMC Complement Altern Med & $3.659(1)$ & 30 & 2.347 \\
\hline 8 & Medicine & Medicine & $1.89(3)$ & 30 & 2.347 \\
\hline 9 & PAIN & Pain & $5.961(1)$ & 30 & 2.347 \\
\hline 10 & Cochrane Database of Systematic Reviews & Cochrane Database Syst Rev & $9.266(1)$ & 28 & 2.191 \\
\hline
\end{tabular}

Note: *IF and Q in category according to Journal Citation Reports (2020).

Abbreviations: IF, impact factor; Q, quartile; LBP, low back pain.

LBP through somatotopically-specific structural S1 neuroplasticity, which was associated with diminished S1-back gray matter volume. ${ }^{34}$

In this study, we provided an insight into acupuncture for LBP research through the bibliometric analysis method between 1985 and 2021. Since the first paper was published in 1992, the publications in this field have been increasing persistently. According to the current trend analysis, the research of acupuncture for LBP will continue to show an overall growth trend in the next few

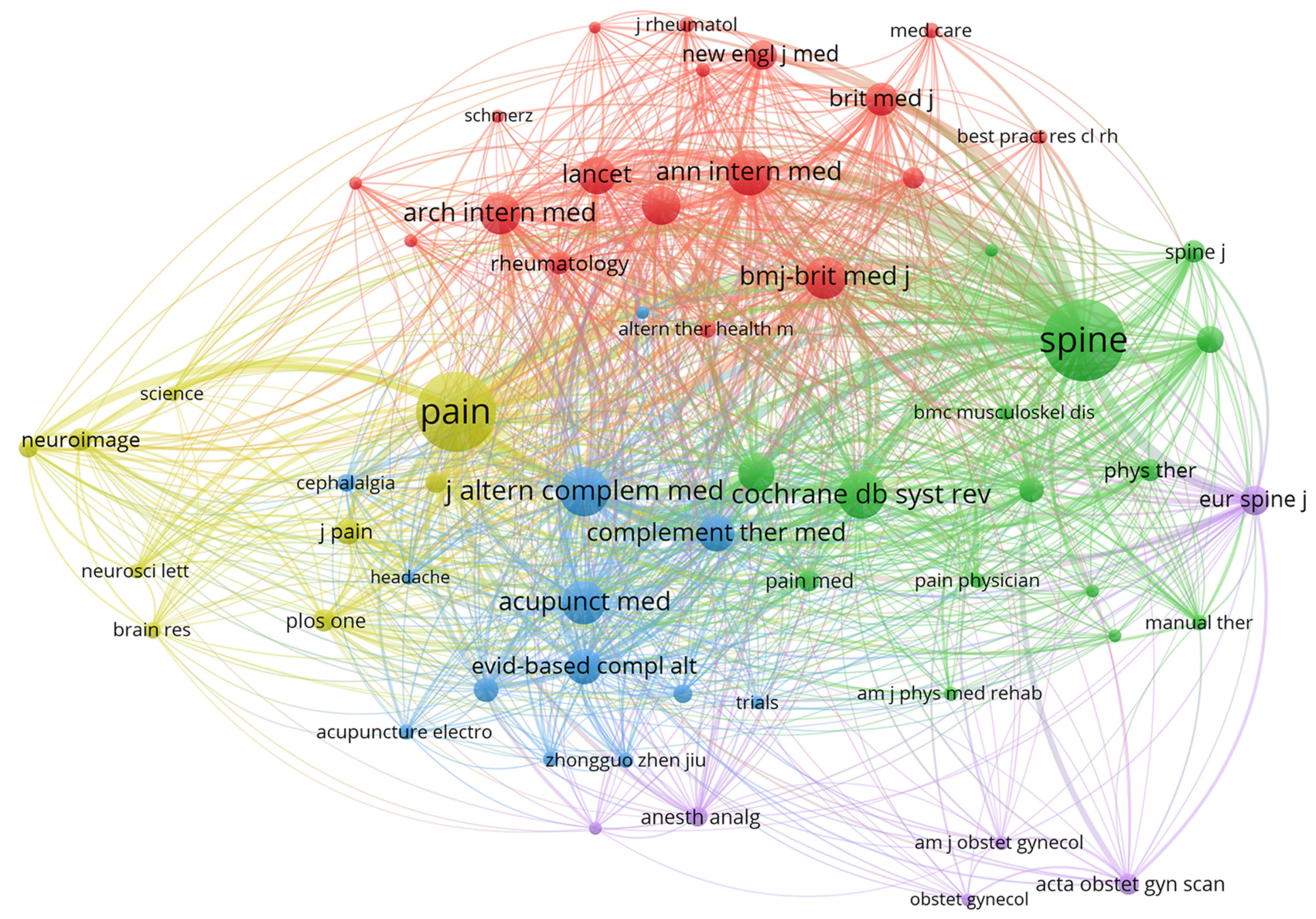

Figure 8 Map of co-cited journals producing publications about acupuncture treatment for LBP. Abbreviation: LBP, low back pain. 
Table 7 Top 10 Co-Cited Journals on Acupuncture for LBP Research

\begin{tabular}{|l|l|l|l|l|}
\hline Ranking & Journal & Abbreviations & Frequency & Centrality \\
\hline 1 & SPINE & Spine & 3111 & 0.02 \\
2 & PAIN & Pain & 2980 & 0.01 \\
3 & Journal of Alternative and Complementary Medicine & J Altern Complem Med & 1334 & 0.02 \\
4 & Cochrane Database of Systematic Reviews & Cochrane Database Syst Rev & 1222 & 1154 \\
5 & Annals of Internal Medicine & Ann Intern Med & 1082 & 0.01 \\
6 & Acupuncture in Medicine & Acupunct Med & 1000 & 0.02 \\
7 & BMJ-British Medical Journal & BMJ-BRIT MED J & 985 & 0.02 \\
8 & Archives of Internal Medicine & Arch Intern Med & 871 & 0.02 \\
9 & Journal of the American Medical Association (JAMA) & JAMA & 858 & 0.01 \\
10 & Lancet & Lancet & 0.01 \\
\hline
\end{tabular}

Abbreviation: LBP, low back pain.

years. The top five productive countries' contributions accounted for $82.41 \%$ of total publications. The centrality analysis indicated that the USA, Peoples R China, and
England were in the dominant positions. Meanwhile, the centrality analysis of the institution was University of Maryland. Furthermore, the collaborations among

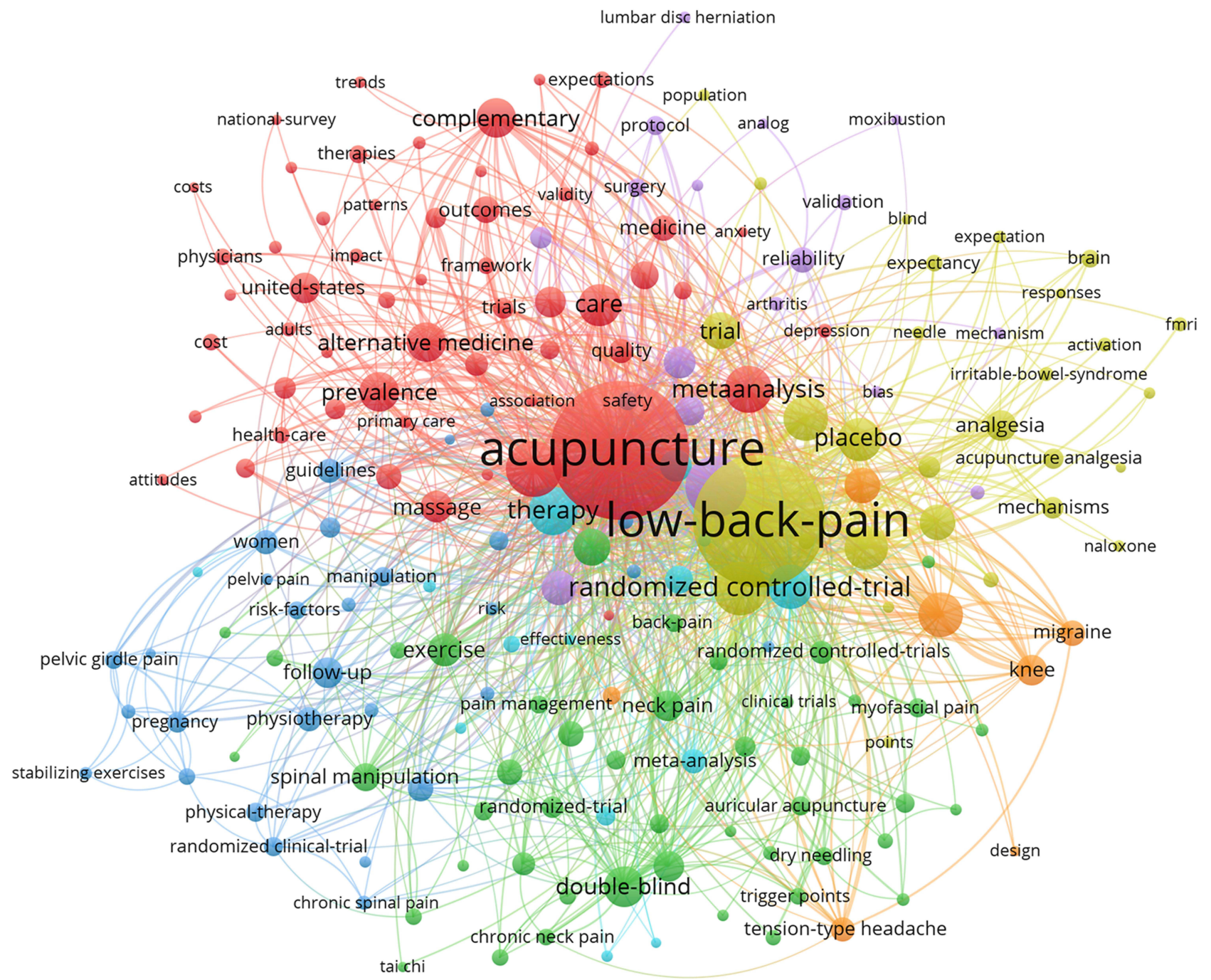

Figure 9 Map of co-occurrence keywords from publications about acupuncture treatment for LBP. Abbreviation: LBP, low back pain. 
Table 8 Top 10 Co-Occurrence Keywords Related to Acupuncture for LBP Research

\begin{tabular}{|l|l|l|l|}
\hline Ranking & Keyword & Frequency & Centrality \\
\hline 1 & Acupuncture & 692 & 0.07 \\
2 & Low-back-pain & 644 & 0.03 \\
3 & Management & 177 & 0.03 \\
4 & Low back pain & 150 & 0.03 \\
5 & Randomized controlled-trial & 142 & 0.08 \\
6 & Therapy & 117 & 0.04 \\
7 & Meta-analysis & 115 & 0.06 \\
8 & Efficacy & 103 & 0.06 \\
9 & Osteoarthritis & 102 & 0.03 \\
10 & Pain & 101 & 0.07 \\
\hline
\end{tabular}

Abbreviation: LBP, low back pain.

institutions showed distinct cluster characteristics, with a relatively long distance among each cluster, which means fewer reciprocal connections exist among them by now, and global cooperation has not yet been formed. Although some western countries still have strong scientific research strength, the focus of research in this field is gradually shifting to the east, especially to Asia in recent years. The network map of author cooperation found that the vast majority of the authors' centralities were equal to
0 , thus reflecting that the lack of cooperation between authors and needed improvement. As for journals, the average impact factor was of the 10 selected journals is 3.65. This result suggested that there is challenging to publish in high-impact journals. One reason for this is that overall the reporting quality of RCTs of acupuncture for LBP was moderate, which did not rigidly follow the CONSORT and STRICTA statements. ${ }^{35}$ Alternatively, high-quality and better-designed researches are needed in the future to be published in high-quality journals gradually, which may help to exchange research findings of acupuncture for LBP. According to the cocitation journal analysis, Spine and Pain were the core journals in the field.

By analyzing the burst keywords, research frontiers could be identified. In this study, the three newest burst keywords are pain management, disease, and protocol. Identifying the disease itself is always the most important thing that researchers focus on. Although the step to investigate the pathologic features, diagnostic procedures, and targeted interventions are continuously moving forwards, much more effort is needed to understand the whole characteristic of LBP. ${ }^{36,37}$ Pain management has been an important part of several guidelines, such as the 2017 ACP clinical practice guideline for chronic low back pain ${ }^{7}$ and the 2021 NICE

Table 9 Top 10 Co-Cited References Related to Acupuncture for LBP

\begin{tabular}{|c|c|c|c|c|}
\hline Ranking & Reference & $\begin{array}{l}\text { Representative Author } \\
\text { (Publication Year) }\end{array}$ & Counts & Centrality \\
\hline I & $\begin{array}{l}\text { German Acupuncture Trials (GERAC) for chronic low back pain: randomized, } \\
\text { multicenter, blinded, parallel-group trial with } 3 \text { groups }\end{array}$ & Haake $(2007)^{19}$ & 186 & 0.01 \\
\hline 2 & Acupuncture in patients with chronic low back pain: a randomized controlled trial & Brinkhaus $(2006)^{21}$ & 164 & 0.05 \\
\hline 3 & $\begin{array}{l}\text { A randomized trial comparing acupuncture, simulated acupuncture, and usual } \\
\text { care for chronic low back pain }\end{array}$ & Cherkin $(2009)^{22}$ & 120 & 0.07 \\
\hline 4 & Meta-analysis: acupuncture for low back pain & Manheimer $(2005)^{23}$ & 118 & 0.07 \\
\hline 5 & $\begin{array}{l}\text { Randomized trial comparing traditional Chinese medical acupuncture, } \\
\text { therapeutic massage, and self-care education for chronic low back pain }\end{array}$ & Cherkin $(200 I)^{24}$ & 108 & 0.07 \\
\hline 6 & Acupuncture for chronic pain: individual patient data meta-analysis & Vickers $(2012)^{25}$ & 105 & 0.02 \\
\hline 7 & $\begin{array}{l}\text { Diagnosis and treatment of low back pain: a joint clinical practice guideline } \\
\text { from the American College of Physicians and the American Pain Society }\end{array}$ & Chou $(2007)^{26}$ & 102 & 0.01 \\
\hline 8 & $\begin{array}{l}\text { Pragmatic randomized trial evaluating the clinical and economic effectiveness of } \\
\text { acupuncture for chronic low back pain }\end{array}$ & Witt $(2006)^{27}$ & 92 & 0.02 \\
\hline 9 & Acupuncture in patients with osteoarthritis of the knee: a randomised trial & Witt $(2005)^{28}$ & 90 & 0.02 \\
\hline 10 & $\begin{array}{l}\text { Acupuncture and dry-needling for low back pain: an updated systematic review } \\
\text { within the framework of the Cochrane collaboration }\end{array}$ & Furlan $(2005)^{20}$ & 85 & 0.23 \\
\hline
\end{tabular}

Abbreviation: LBP, low back pain. 


\begin{tabular}{lccccll}
\hline \multicolumn{1}{c}{ Keywords } & Year & Strength & Begin & End & & 1992 - 2021 \\
\hline rheumatoid arthriti & 1992 & 5 & $\mathbf{1 9 9 2}$ & 2003 & \\
controlled trial & 1992 & 11.42 & $\mathbf{1 9 9 5}$ & 2005 & \\
cost & 1992 & 4.73 & $\mathbf{1 9 9 7}$ & 2006 & \\
spinal manipulation & 1992 & 4.65 & $\mathbf{1 9 9 7}$ & 2008 & \\
follow up & 1992 & 4.93 & $\mathbf{1 9 9 9}$ & 2005 & \\
massage & 1992 & 7.22 & $\mathbf{2 0 0 2}$ & 2007 & \\
electrical nerve & 1992 & 4.49 & $\mathbf{2 0 0 2}$ & 2007 & \\
stimulation & & & & & \\
placebo controlled trial & 1992 & 5.67 & $\mathbf{2 0 0 4}$ & 2008 & \\
knee & 1992 & 6.69 & $\mathbf{2 0 0 6}$ & 2012 & \\
migraine & 1992 & 4.67 & $\mathbf{2 0 0 6}$ & 2012 & \\
placebo & 1992 & 7.05 & $\mathbf{2 0 0 7}$ & 2010 & \\
framework & 1992 & 5.62 & $\mathbf{2 0 1 0}$ & 2014 & \\
mechanism & 1992 & 5.11 & $\mathbf{2 0 1 1}$ & 2017 & \\
comparing acupuncture & 1992 & 4.74 & $\mathbf{2 0 1 2}$ & 2016 & \\
intervention & 1992 & 4.39 & $\mathbf{2 0 1 4}$ & 2016 & \\
physiotherapy & 1992 & 6.21 & $\mathbf{2 0 1 5}$ & 2017 & \\
chronic pain & 1992 & 5.06 & $\mathbf{2 0 1 5}$ & 2019 & \\
pain management & 1992 & 4.74 & $\mathbf{2 0 1 5}$ & 2021 & \\
disease & 1992 & 5.11 & $\mathbf{2 0 1 8}$ & 2021 & \\
protocol & 1992 & 6.56 & $\mathbf{2 0 1 9}$ & 2021 & \\
\hline
\end{tabular}

Figure 10 Top 20 keywords with the strongest citation bursts.

guideline for chronic pain (NG193, www.nice.org.uk/gui dance/ng193). Briefly, the patients with acute or subacute LBP are recommended to select nonpharmacologic treatment including acupuncture, or pharmacologic treatment such as nonsteroidal anti-inflammatory drugs or skeletal-muscle relaxants if the pharmacologic treatment is failed; the patients with chronic LBP are suggested to initially select nonpharmacologic treatment with exercise, acupuncture, yoga, and others. ${ }^{7}$ In recent years, the self-management of patients has also been greater emphasized. ${ }^{38}$ The protocols focusing on clinical study ${ }^{31,39-41}$ or systematic review and metaanalysis $^{42-46}$ are increasing within these years, which suggests that more attention has been paid to this field to elucidate the potential mechanisms of acupuncture for LBP.

Though we have to acknowledge that there is an overlap between our study and other previous studies, ${ }^{12,47,48}$ the present research makes some novel contributions to this field. (1) We included the papers published in the recent 5 years to complement and enrich our results; (2) we combine the two analysis tools, VOSviewer and CiteSpace, to present the results comprehensively. However, this study also has some limitations. (1) We only include publications with the English language, leaving other languages not to analyze; (2) the VOSviewer might not differ associated organizations precisely, for example, the Harvard Medical School and Harvard University.

\section{Conclusion}

LBP has a prevalence of approximately $7 \%$ around the world and is common to recurrent. Non-pharmacological approaches have been regarded as first-line treatments for LBP, and acupuncture has also been recommended by guidelines. In this bibliometric analysis research, the results suggested the present situation of the publications in the field of acupuncture for LBP, as well as the hot spots and research frontiers in this field. The research of acupuncture for LBP still has great development potential and much more international communications and cooperation as well as studies with good design are needed in the future.

\section{Ethics Approval}

This article does not contain any studies with human or animal subjects performed by any of the authors.

\section{Funding}

The project was funded by the National Natural Science Fund of China (81973940), Accelerating the Development of Chinese Medicine Three-Year Action Plan of Shanghai (No. ZY (2018-2020)-CCCX-2004-04), Clinical Key Specialty Construction Foundation of Shanghai (No. shslczdzk04701), and Shanghai Clinical Research Center for Acupuncture and Moxibustion (20MC1920500). 


\section{Disclosure}

The authors declare that they have no conflicts of interest.

\section{References}

1. Lewis K, Abdi S. Acupuncture for lower back pain: a review. Clin J Pain. 2010;26(1):60-69. doi:10.1097/AJP.0b013e3181bad71e

2. Ma Y, Dong M, Zhou K, Mita C, Liu J, Wayne PM. Publication trends in acupuncture research: a 20-year bibliometric analysis based on PubMed. PLoS One. 2016;11(12):e0168123. doi:10.1371/journal. pone. 0168123

3. Lee I-S, Chae Y. A bibliometric analysis of acupuncture research trends in. Med Acupunct Med. 2019;37(6):375-377. doi:10.1177/ 0964528419884327

4. Treede RD, Rief W, Barke A, et al. Chronic pain as a symptom or a disease: the IASP classification of chronic pain for the International Classification of Diseases (ICD-11). Pain. 2019;160(1):19-27. doi:10.1097/j.pain.0000000000001384

5. Hartvigsen J, Hancock MJ, Kongsted A, et al. What low back pain is and why we need to pay attention. Lancet. 2018;391(10 137):2356-2367. doi:10.1016/S0140-6736(18)30480-X

6. Dionne CE, Dunn KM, Croft PR, et al. A consensus approach toward the standardization of back pain definitions for use in prevalence studies. Spine. 2008;33(1):95-103. doi:10.1097/BRS.0b013e31815 e7f94

7. Qaseem A, Wilt TJ, McLean RM, Forciea MA. Noninvasive treatments for acute, subacute, and chronic low back pain: a clinical practice guideline from the American College of Physicians. Ann Intern Med. 2017;166(7):514-530. doi:10.7326/M16-2367

8. Dowell D, Haegerich TM, Chou R. CDC guideline for prescribing opioids for chronic pain-United States, 2016. JAMA. 2016;315 (15):1624-1645. doi:10.1001/jama.2016.1464

9. Bogduk N. On the definitions and physiology of back pain, referred pain, and radicular pain. Pain. 2009;147(1-3):17-19. doi:10.1016/j. pain.2009.08.020

10. da Silva T, Mills K, Brown BT, et al. Recurrence of low back pain is common: a prospective inception cohort study. J Physiother. 2019;65 (3):159-165. doi:10.1016/j.jphys.2019.04.010

11. Knezevic NN, Candido KD, Vlaeyen JWS, Van Zundert J, Cohen SP. Low back pain. Lancet. 2021;398:78-92. doi:10.1016/S0140-673 6(21)00733-9

12. Lee I-S, Lee H, Chen Y-H, Chae Y. Bibliometric analysis of research assessing the use of acupuncture for pain treatment over the past 20 years. J Pain Res. 2020;13:367-376. doi:10.2147/JPR.S235047

13. Zhang J, Zhang Y, Hu L, et al. Global trends and performances of magnetic resonance imaging studies on acupuncture: a bibliometric analysis. Front Neurosci. 2020;14:620555. doi:10.3389/fnins.2020. 620555

14. van Eck NJ, Waltman L. Software survey: vOSviewer, a computer program for bibliometric mapping. Scientometrics. 2010;84 (2):523-538. doi:10.1007/s11192-009-0146-3

15. van Eck NJ, Waltman L. Citation-based clustering of publications using CitNetExplorer and VOSviewer. Scientometrics. 2017;111 (2):1053-1070. doi:10.1007/s11192-017-2300-7

16. Chen C. Searching for intellectual turning points: progressive knowledge domain visualization. Proc Natl Acad Sci U S A. 2004;101 (Suppl 1):5303-5310. doi:10.1073/pnas.0307513100

17. Zhang Y, Li C, Ji X, Yun C, Wang M, Luo X. The knowledge domain and emerging trends in phytoremediation: a scientometric analysis with CiteSpace. Environ Sci Pollut Res Int. 2020;27(13):1551 5-15536. doi:10.1007/s11356-020-07646-2

18. MacPherson H, Thorpe L, Thomas K, Campbell M. Acupuncture for low back pain: traditional diagnosis and treatment of 148 patients in a clinical trial. Complement Ther Med. 2004;12(1):38-44. doi:10. 1016/S0965-2299(03)00125-0
19. Haake M, Müller-H-H, Schade-Brittinger C, et al. German Acupuncture Trials (GERAC) for chronic low back pain: randomized, multicenter, blinded, parallel-group trial with 3 groups. Arch Intern Med. 2007;167 (17):1892-1898. doi:10.1001/Archinte.167.17.1892

20. Furlan AD, van Tulder M, Cherkin D, et al. Acupuncture and dry-needling for low back pain: an updated systematic review within the framework of the Cochrane collaboration. Spine. 2005;30 (8):944-963. doi:10.1097/01.brs.0000158941.21571.01

21. Brinkhaus B, Witt CM, Jena S, et al. Acupuncture in patients with chronic low back pain: a randomized controlled trial. Arch Intern Med. 2006;166(4):450-457.

22. Cherkin DC, Sherman KJ, Avins AL, et al. A randomized trial comparing acupuncture, simulated acupuncture, and usual care for chronic low back pain. Arch Intern Med. 2009;169(9):858-866.

23. Manheimer E, White A, Berman B, Forys K, Ernst E. Meta-analysis: acupuncture for low back pain. Ann Intern Med. 2005;142 (8):651-663.

24. Cherkin DC, Eisenberg D, Sherman KJ, et al. Cherkin DC, Eisenberg $\mathrm{D}$, Sherman $\mathrm{KJ}$, et al. Randomized trial comparing traditional Chinese medical acupuncture, therapeutic massage, and self-care education for chronic low back pain. Arch Intern Med. 2001;161 (8):1081-1088.

25. Vickers AJ, Cronin AM, Maschino AC, et al. Acupuncture for chronic pain: individual patient data meta-analysis. Arch Intern Med. 2012;172(19):1444-1453.

26. Chou R, Qaseem A, Snow V, et all. Diagnosis and treatment of low back pain: a joint clinical practice guideline from the American College of Physicians and the American Pain Society. Ann Intern Med. 2007;147(7):478-491.

27. Witt CM, Jena S, Selim D, et al. Pragmatic randomized trial evaluating the clinical and economic effectiveness of acupuncture for chronic low back pain. Am J Epidemiol. 2006;164(5):487-496.

28. Witt C, Brinkhaus B, Jena S, et al. Acupuncture in patients with osteoarthritis of the knee: a randomised trial. Lancet (London, England). 2005;366(9480):136-143.

29. Fuentes RC, Organ B, Creech J, Broszko CM, Nashelsky J. Acupuncture for low back pain. Can Fam Physician. 2020;66 (3):186-187.

30. Yeganeh M, Baradaran HR, Qorbani M, Moradi Y, Dastgiri S. The effectiveness of acupuncture, acupressure and chiropractic interventions on treatment of chronic nonspecific low back pain in Iran: a systematic review and meta-analysis. Complement Ther Clin Pract. 2017;27:11-18. doi:10.1016/j.ctcp.2016.11.012

31. Wei X, Liu B, He L, et al. Acupuncture therapy for chronic low back pain: protocol of a prospective, multi-center, registry study. $B M C$ Musculoskelet Disord. 2019;20(1):488.

32. Wang L, Yin Z, Zhang Y, et al. Optimal acupuncture methods for nonspecific low back pain: a systematic review and bayesian network meta-analysis of randomized controlled trials. $J$ Pain Res. 2021;14:1097-1112. doi:10.2147/JPR.S310385

33. Wen Q, Ma P, Dong X, et al. Neuroimaging studies of acupuncture on low back pain: a systematic review. Front Neurosci. 2021;15:730322. doi:10.3389/fnins.2021.730322

34. Kim H, Mawla I, Lee J, et al. Reduced tactile acuity in chronic low back pain is linked with structural neuroplasticity in primary somatosensory cortex and is modulated by acupuncture therapy. NeuroImage. 2020;217:116899. doi:10.1016/j.neuroimage.2020.116899

35. Liu X, Xu Z, Wang Y, et al. Evaluating the quality of reports about randomized controlled trials of acupuncture for low back pain. J Pain Res. 2021;14:1141-1151. doi:10.2147/JPR.S308006

36. Vlaeyen JWS, Maher CG, Wiech K, et al. Low back pain. Nat Rev Dis Primers. 2018;4(1):52. doi:10.1038/s41572-018-0052-1

37. Maher C, Underwood M, Buchbinder R. Non-specific low back pain. Lancet. 2017;389(10070):736-747. doi:10.1016/S0140-6736(16) 30970-9 
38. Foster NE, Anema JR, Cherkin D, et al. Prevention and treatment of low back pain: evidence, challenges, and promising directions. Lancet. 2018;391(10137):2368-2383. doi:10.1016/S0140-6736(18) 30489-6

39. Kim JH, Na CS, Park GC, Lee JS. Effects of different wavelengths of invasive laser acupuncture on chronic non-specific low back pain: a study protocol for a pilot randomized controlled trial. Trials. 2021;22(1):118. doi:10.1186/s13063-021-05038-6

40. Li T, Wang S, Zhang S, et al. Evaluation of clinical efficacy of silver-needle warm acupuncture in treating adults with acute low back pain due to lumbosacral disc herniation: study protocol for a randomized controlled trial. Trials. 2019;20(1):470.

41. Francescato Torres S, Brandt de Macedo AC, Dias Antunes M, et al. Effects of electroacupuncture frequencies on chronic low back pain in older adults: triple-blind, 12-months protocol for a randomized controlled trial. Trials. 2019;20(1):762. doi:10.1186/s13063-019-3813-6

42. Zhong X, Liu J, Wang Y, Zhang L, Zhang H. Which of the acupuncture treatment regimen for lumbar disc herniation is more effective and safer: a protocol for systematic review and network meta-analysis. Medicine. 2021;100(12):e25199. doi:10.1097/MD.00 00000000025199
43. Yuan S, Huang C, Xu Y, Chen D, Chen L. Acupuncture for lumbar disc herniation: protocol for a systematic review and meta-analysis. Medicine. 2020;99(9):e19117. doi:10.1097/MD.0000000000019117

44. Deng R, Huang Z, Li X, Pei X, Li C, Zhao J. The effectiveness and safety of acupuncture in the treatment of lumbar disc herniation: protocol for a systematic review and meta-analysis. Medicine. 2020;99(12):e18930. doi:10.1097/MD.0000000000018930

45. Liao J, Wang T, Dong W, et al. Acupoint injection for nonspecific chronic low back pain: a protocol of systematic review. Medicine. 2019;98(29):e16478. doi:10.1097/MD.0000000000016478

46. Chen Y, Li X, Xu J, Chen J, Huo Z, Zhang L. Acupuncture for lumbar myofascial pain protocol for a systematic review of randomized controlled trials. Medicine. 2019;98(26):e16271. doi:10.1097/ MD.0000000000016271

47. Gao Z, Zhang J, Liu G-F, Ji L-X. Research trends from 2010 to 2020 for pain treatment with acupuncture: a bibliometric analysis. $J$ Pain Res. 2021;14:941-952. doi:10.2147/JPR.S300911

48. Liang Y-D, Li Y, Zhao J, Wang X-Y, Zhu H-Z, Chen X-H. Study of acupuncture for low back pain in recent 20 years: a bibliometric analysis via CiteSpace. J Pain Res. 2017;10:951-964. doi:10.2147/ JPR.S132808

\section{Publish your work in this journal}

The Journal of Pain Research is an international, peer reviewed, open access, online journal that welcomes laboratory and clinical findings in the fields of pain research and the prevention and management of pain. Original research, reviews, symposium reports, hypothesis formation and commentaries are all considered for publication. The manuscript management system is completely online and includes a very quick and fair peer-review system, which is all easy to use. Visit http:// www.dovepress.com/testimonials.php to read real quotes from published authors. 